\title{
Estimation of mineral and trace element intake in vegans living in Japan by chemical analysis of duplicate diets
}

\author{
Munehiro Yoshida*, Noriko Ôgi, Yuki Iwashita
}

Laboratory of Food and Nutritional Sciences, Faculty of Chemistry, Materials and Bioengineering, Kansai University, Suita, Japan;

*Corresponding Author: hanmyou4@kansai-u.ac.jp

Received 10 September 2011; Revised 5 October 2011; Accepted 21 October 2011.

\begin{abstract}
Thirty-six daily duplicate diet samples were collected from 12 healthy female Japanese vegans and sodium, potassium, calcium, magnesium, phosphorus, iron, zinc, copper, manganese, iodine, selenium, chromium and molybdenum in the diets were measured to estimate mineral and trace element intake by Japanese vegans. Significantly higher intake of potassium, magnesium, phosphorus, iron, copper, manganese and molybdenum was observed in vegans than in general Japanese women, but no difference was observed in sodium, iodine, selenium and chromium intake. Vegan calcium intake tended to be low compared to that of general women but the difference was not significant. Since high potassium, magnesium and iron intakes cannot be achieved by general Japanese diets and high intake of potassium and magnesium may prevent hyperextension and cardiovascular disease in vegans, there are few problems with Japanese vegan diets regarding mineral and trace element intake, except for calcium intake, which is low as it is in the general Japanese people.
\end{abstract}

Keywords: Vegan; Mineral intake; Trace Element Intake; Duplicate Diets; Japan

\section{INTRODUCTION}

Vegetarian diets, essentially excluding animal foods, have become increasingly popular in developed countries [1]. These diets are classified according to the types of animal foods consumed, and strict vegetarians consuming no foods of animal origin are known as vegans. Although vegan diets cause lower serum cholesterol, lower blood pressure and a reduced risk of cardiovascular diseases, eliminating all animal foods from the diet increases the risk of several micronutrient deficiencies, including vitamin $\mathrm{B}_{12}$, vitamin $\mathrm{D}$ and $\mathrm{n}-3$ fatty acids [2]. Regarding the intake of minerals and trace elements, vegetarians, including vegans, show low intakes of calcium, zinc and selenium because the main sources of these micronutrients are animal foods in Western diets $[3,4]$.

Traditional Asian diets are predominately plant-based, differing from Western diets. In Japan, although the consumption of meat and dairy products has increased along with the Westernization of society, more than three quarters of the energy intake still depends on plant foods [5]. Accordingly, it is thought that the effect of adopting a vegan diet on the nutrient intake pattern is different between the West and Japan. However, little research has examined the nutrient intake of vegetarians in Japan [6], and research on the intake of minerals and trace elements by Japanese vegans is scarce. In the present study, to evaluate mineral and trace element intake by Japanese vegans, duplicate diet samples were collected from Japanese vegans, and concentrations of sodium, potassium, calcium, magnesium, phosphorus, iron, zinc, copper, manganese, iodine, selenium, chromium and molybdenum were measured.

\section{SUBJECTS AND METHODS}

\subsection{Subjects and Duplicate Diet Sampling}

In the present study, vegans were defined as people eating food of plant origin only. Twelve healthy female vegans were recruited through a vegetarian food shop located in Chiba Prefecture, Japan. The characteristics of the subjects are described in Table 1. Duplicate meals, beverages and between-meal snacks were collected over 24 h period; 36 duplicate diets from 12 subjects were sampled for 3 consecutive days between September and November 2010. All subjects gave informed consent for the use of their personal information in this study. 
Table 1. Characteristics of vegan subjects $(n=12)$.

\begin{tabular}{lcc}
\hline & Mean \pm SD & Median \\
\hline Age (y) & $48.4 \pm 12.9$ & 47.5 \\
Duration of vegan diet (y) & $20.7 \pm 14.5$ & 12.0 \\
Height $(\mathrm{cm})$ & $156.4 \pm 7.7$ & 157.0 \\
Weight $(\mathrm{kg})$ & $49.1 \pm 8.9$ & 48.5 \\
Body mass index $\left(\mathrm{kg} / \mathrm{m}^{2}\right)$ & $19.9 \pm 2.4$ & 19.7 \\
\hline
\end{tabular}

\subsection{Treatment of Samples}

The daily duplicate diet sample was freeze-dried, homogenized and milled. Approximately $1 \mathrm{~g}$ of the dried sample was mixed with $200 \mathrm{~mL}$ of $1 \% \mathrm{HCl}$, shaken for 30 min and centrifuged. The supernatant was filtrated with $0.45-\mu \mathrm{m}$ membrane filter. Filtrate thus obtained was used for the determination of sodium and potassium. Another $1 \mathrm{~g}$ of the dried sample was heated with $10 \mathrm{~mL}$ metal-free $\mathrm{HNO}_{3}$ until the disappearance of insoluble components, and then, $2 \mathrm{~mL}$ metal-free $\mathrm{HClO}_{4}$ was added to the digestion mixture, which was further heated until the appearance of white vapor of $\mathrm{HClO}_{4}$. The volume of the digest was made up to $10 \mathrm{~mL}$ with pure water. Diluted digest thus obtained was used for the determination of calcium, magnesium, phosphorus, iron, zinc, copper, manganese, selenium and molybdenum. For the analysis of chromium, approximately $1 \mathrm{~g}$ of the dried sample was heated in an electric furnace (F-B1414M; As One, Osaka, Japan) at $550^{\circ} \mathrm{C}$ for 16 h [7]. After dry incineration, the remaining ash was dissolved in $10 \mathrm{~mL}$ of $0.1 \mathrm{M} \mathrm{HNO}_{3}$. Iodine in the dried samples was extracted with $0.5 \%$ tetramethylammonium hydroxide (TMAH) [8]. Two hundred milligrams of the dried samples was mixed with $40 \mathrm{~mL}$ of $0.5 \%$ TMAH and left overnight. The mixture was heated at $60^{\circ} \mathrm{C}$ for $6 \mathrm{~h}$ and centrifuged. The supernatant was filtrated through a $0.45-\mu \mathrm{m}$ membrane filter.

\subsection{Analysis}

Sodium, potassium, calcium, magnesium, iron, zinc, copper and manganese were measured using atomic absorption spectrometer (AA-6300; Shimadzu, Kyoto, Japan). Iodine, selenium, chromium and molybdenum were determined by inductively coupled plasma mass spectrometry (ICPMS) with direct nebulization. The ICPMS operating conditions were as follows: instrument, ICPM8500 (Shimadzu); forward power, $1200 \mathrm{~W}$; coolant gas flow rate, $7.0 \mathrm{~L} / \mathrm{min}$; auxiliary gas flow rate, $1.5 \mathrm{~L} / \mathrm{min}$; nebulizer gas flow rate, $0.58 \mathrm{~L} / \mathrm{min}$; sampling depth, 5.0 $\mathrm{mm}$; integration time, $2.0 \mathrm{~s}$; number of run, 20; mode of analysis, pulse; isotopes monitored, ${ }^{52} \mathrm{Cr},{ }^{82} \mathrm{Se},{ }^{95} \mathrm{Mo}$, ${ }^{97} \mathrm{Mo},{ }^{98} \mathrm{Mo}$ and ${ }^{127} \mathrm{I}$. Rhodium $\left({ }^{103} \mathrm{Rh}\right)$ and tellurium $\left({ }^{126} \mathrm{Te},{ }^{128} \mathrm{Te}\right.$ and $\left.{ }^{130} \mathrm{Te}\right)$ were used as internal standards. Phosphorus was determined with vanadomolybdate absorption spectrometry [9]. Protein, total lipid and energy were analyzed by a commercial service system (Japan Functional Food Analysis and Research Center, Fukuoka, Japan).

\subsection{Statistical Analysis}

For each subject, mean daily intake was calculated from the analytical results of duplicate diet samples from 3 consecutive days. The mean and median of the daily intake for 12 subjects were then calculated. For iodine, the mean and median were also calculated when each value was logarithmically transformed because values highly varied. Mean daily intake for 12 subjects was statistically compared with the mean daily intake by general Japanese women aged 30 to $49 \mathrm{y}$ described in the National Health and Nutritional Survey in Japan (NHNSJ) [10] by calculation of the Z-score; in which women aged 30 to $49 \mathrm{y}$ in NHNSJ, $2008(\mathrm{n}=1053)$ were regarded as a population.

\section{RESULTS AND DISCUSSION}

In Table 2, daily intake of major nutrients, minerals and trace elements by 12 Japanese female vegans was summarized and compared with those by general Japanese women and several criteria in the Dietary Reference Intakes for Japanese (DRIJ) [11]. For the intake of energy, protein and total lipids, no difference was observed between vegans and general women.

Among major mineral intake, calcium intake by vegans was below the estimated average requirement (EAR) and tended to be low compared to that by the general population. In several Western researches, calcium intake by vegans was markedly lower than that by omnivores [12] and lacto-vegetarians [13]. In the present analysis, vegan calcium intake was somewhat low but was not significantly lower than in the general Japanese calcium intake. Since calcium intake by general Japanese people is always low due to the low consumption of dairy products, the low calcium intake of Japanese vegans may be inconspicuous.

Phosphorus intake by vegans was markedly higher than by general women. In Western research, a vegan diet contains low phosphorus and is appropriate for patients with renal failure [14]. In the West, because the major source of phosphorus in general diets is dairy products, vegan phosphorus intake is comparatively low; however, Japanese people ingest phosphorus mainly from plant foods [5]. The difference in the source of 
Table 2. Intake of energy, protein, lipids, minerals and trace elements in Japanese vegans.

\begin{tabular}{|c|c|c|c|c|c|c|c|c|c|}
\hline & \multicolumn{2}{|c|}{ Vegans $(\mathrm{n}=12)$} & \multicolumn{2}{|c|}{ NHNSJ, 2008 } & \multicolumn{5}{|c|}{ DRIJ, 2010²) } \\
\hline & Mean \pm SD & Median & Mean \pm SD & Median & EAR & RDA & AI & DG & UL \\
\hline Energy (kcal) & $1847 \pm 141$ & 1840 & $1682 \pm 469$ & 1645 & 1750 & - & - & - & - \\
\hline Protein (g) & $56.2 \pm 8.1$ & 58.4 & $60.2 \pm 19.0$ & 58.7 & 40 & 50 & - & - & - \\
\hline Lipids (\% energy) & $20.8 \pm 7.3$ & 21.0 & $24.5 \pm 14.1$ & 22.6 & - & - & - & $20-25$ & - \\
\hline Sodium (mg) & $3649 \pm 1719$ & 3029 & $3696 \pm 1415^{3)}$ & $3538^{3)}$ & $590^{3)}$ & - & - & $<2950^{3)}$ & - \\
\hline Potassium (mg) & $3610 \pm 1272 *$ & 3217 & $1983 \pm 777$ & 1891 & - & - & 2000 & 2800 & - \\
\hline Calcium (mg) & $361 \pm 122$ & 389 & $440 \pm 224$ & 406 & 550 & 650 & - & - & 2300 \\
\hline Magnesium (mg) & $494 \pm 112^{*}$ & 462 & $214 \pm 80$ & 204 & 240 & 290 & - & - & - \\
\hline Phosphorus (mg) & $1225 \pm 311^{*}$ & 1197 & $854 \pm 284$ & 830 & - & - & - & - & 3000 \\
\hline Iron (mg) & $13.0 \pm 2.4^{*}$ & 12.2 & $6.9 \pm 3.0$ & 6.5 & 9.0 & 11.0 & 900 & - & 40 \\
\hline Zinc (mg) & $8.3 \pm 1.6$ & 9.1 & $7.1 \pm 2.4$ & 6.9 & 8 & 9 & - & - & 35 \\
\hline Copper (mg) & $1.75 \pm 0.37^{*}$ & 1.66 & $1.00 \pm 0.35$ & 0.96 & 0.6 & 0.7 & - & - & 10 \\
\hline Manganese (mg) & $7.5 \pm 2.2$ & 7.9 & - & - & - & - & 3.5 & - & 11 \\
\hline \multirow[t]{2}{*}{ Iodine ( $\mu \mathrm{g}$ ) } & $1865 \pm 1934$ & 1158 & - & - & 95 & 130 & - & - & 2200 \\
\hline & $788(255-2441)^{4)}$ & $746^{5)}$ & & & & & & & \\
\hline Selenium $(\mu \mathrm{g})$ & $87 \pm 34$ & 76 & - & - & 20 & 25 & - & - & 230 \\
\hline Chromium ( $\mu \mathrm{g})$ & $27 \pm 8$ & 28 & - & - & 25 & 30 & - & - & - \\
\hline Molybdenum ( $\mu \mathrm{g})$ & $540 \pm 207$ & 563 & - & - & 20 & 25 & - & - & 500 \\
\hline
\end{tabular}

*Significant difference from NHNSJ data was observed at $p<0.001$ by calculation of $Z$-score; ${ }^{1)}$ Values for general Japanese women aged 30 to 49 y (n = 1053) quoted from the National Health and Nutrition Survey in Japan, 2008 [10]; ${ }^{2}$ Criteria for Japanese women aged 30 to 49 y in Dietary Reference Intakes for Japanese, 2010 [11]; EAR, estimated average requirement; RDA, recommended dietary allowance; AI, adequate intake; DG, tentative dietary goal for preventing lifestyle-related diseases; UL, tolerable upper intake level; ${ }^{3)}$ Calculated from the values for salt; ${ }^{4)}$ Geometrical mean with SD range in parentheses; ${ }^{5)}$ Median calculated after logarithmic transformation of data for each daily duplicate diet sample.

phosphorus may contribute to the difference in phosphorus intake between Western and Japanese vegans. In addition, phytate may contribute to the high phosphorus intake in vegans because whole grains and beans contain it at a high level.

No difference was observed between vegans and general women in sodium intake. On the other hand, vegan potassium intake was markedly higher than by general women and far exceeded the tentative dietary goal for preventing lifestyle-related diseases (DG) in DRIJ. Similarly, markedly higher magnesium intake was observed in vegans than in general women. This high intake of potassium and magnesium is probably due to the high consumption of vegetables and fruit.

Among trace element intake, significantly higher iron and copper intake was observed in vegans than in general women. Similarly, manganese and molybdenum intake by vegans was markedly higher than by general Japanese, as described in several reports $[15,16]$. Intake of these four trace elements far exceeded the recom- mended dietary allowance (RDA) or the adequate intake (AI) in DRIJ. High intake of copper and manganese is also reported in Western researches [17], probably, because the high consumption of whole grains and beans results in high intake of these trace elements. The mean and median of vegan molybdenum intake exceeded the tolerable upper intake level of this element in DRIJ. This is also caused by high consumption of cereals and beans since they particularly soybean, contain molybdenum at a high level [16].

Although vegan zinc intake has been reported to be low [12], there was no difference between vegans and general women; however, because it has been reported that the serum zinc level in Japanese vegetarians tends to be low [18], it is necessary to examine whether phytate and/or dietary fiber, which are contained in whole grains and beans at a high level, decrease the bioavailability of zinc in Japanese vegan diets.

Since the main sources of selenium in general Japanese diets are fish, meats and eggs [19], the low sele- 
nium intake by Japanese vegans is concern; however, selenium intake by Japanese vegans was comparable to that by general Japanese described in several previous reports [19-21]. Japanese vegans may ingest selenium from imported wheat and soybeans, which contain selenium at a high level [22]. Similarly to selenium intake, iodine and chromium intake by vegans was also comparable to general Japanese people described in the literature [20,23].

In conclusion, Japanese vegans are estimated to ingest high potassium, magnesium, phosphorus, iron, copper, manganese and molybdenum compared to general Japanese people. In particular, high potassium, magnesium and iron intake cannot be achieved by ingesting general Japanese diets. High intake of potassium and magnesium may lead to the preventing of hyperextension and cardiovascular disease in vegans [24]. Accordingly, there are few problems with Japanese vegan diets regarding mineral and trace element intake, except for calcium intake, which is low as it is in general Japanese people.

\section{ACKNOWLEDGEMENTS}

This study was supported by a grant for comprehensive research on cardiovascular and lifestyle disease from the Ministry of Health, Labour and Welfare of Japan.

\section{REFERENCES}

[1] Dwyer, J.T. (1991) Nutritional consequences of vegetarianisms. Annual Review of Nutrition, 11, 61-91. doi:10.1146/annurev.nu.11.070191.000425

[2] Craig, W.T. (2009) Health effects of vegan diets. American Journal of Clinical Nutrition, 89, 1627S-1633S. doi:10.3945/ajcn.2009.26736N

[3] Freeland-Graves, J. (1988) Mineral adequacy of vegetarian diets. American Journal of Clinical Nutrition, 48, 859862.

[4] Gibson, R.S. (1994) Content and bioavailability of trace elements in vegetarian diets. American Journal of Clinical Nutrition, 59, 1223S-1232S.

[5] Ministry of Health, Labour and Welfare, Japan (2011) Outline of the National Health and Nutritional Survey of Japan in 2008, 106-165 (in Japanese), Dai-ichi Shuppan, Tokyo.

[6] Nakamoto, K., Watanabe, S., Kudo, H. and Tanaka, A. (2008) Nutritional characteristics of middle-aged Japanese vegetarians. Journal of Atherosclerosis and Thrombosis, 15, 122-129. doi:10.5551/jat.E546

[7] Yoshida, M., Takada, A., Hirose, J., Endô, M., Fukuwatari, T. and Shibata K. (2008) Molybdenum and chromium concentrations in breast milk from Japanese women. Bioscience Biotechnology and Biochemistry, 72, 2247-2250. doi:10.1271/bbb.80283

[8] Dawczynski, C., Schäfer, U., Leiterer, M. and Jahreis, G. (2007) Nutritional and toxicological importance of macro, trace, and ultra-trace elements in algae food products. Journal of Agricultural and Food Chemistry, 55, 10470-
10475. doi:10.1021/if0721500

[9] Quinlan, K.P. and De Sesa, M.A. (1955) Spectrophotometric determination of phosphorus as molybdovanadophosphoric acid. Analytical Chemistry, 27, 16261629. doi:10.1021/ac60106a039

[10] Ministry of Health, Labour and Welfare, Japan (2011) Outline of the National Health and Nutritional Survey of Japan in 2008, 309-329 (in Japanese), Dai-ichi Shuppan, Tokyo.

[11] Ministry of Health, Labour and Welfare, Japan (2009) Report of the Dietary Reference Intakes for Japanese in 2010, 189-275 (in Japanese), National Printing Bureau, Tokyo.

[12] Larsson, C.L. and Johansson, G.K. (2002) Dietary intake and nutritional status of young vegans and omnivores in Sweden. American Journal of Clinical Nutrition, 76, 100-106.

[13] Kramer, L.B., Osis, D., Coffey, J. and Spencer, H. (1984) Mineral and trace element content of vegetarian diets. Journal of American College of Nutrition, 3, 3-11.

[14] Barsotti, G., Morelli, E., Cupisti, A., Meola, M., Dani, L. and Giovannetti, S. (1996) A low-nitrogen low-phosphorus vegan diet for patients with chronic renal failure. Nephron, 74, 390-394. doi:10.1159/000189341

[15] Horiguchi, S., Teramoto, K., Kurono, T. and Ninomiya, K. (1978) The arsenic, copper, lead, manganese and zinc contents of daily foods and beverages in Japan and the estimate of their daily intake. Osaka City Medical Journal, 24, 131-141.

[16] Hattori, H., Ashida, A., Itô, C. and Yoshida, M. (2004) Determination of molybdenum in foods and human milk, and an estimate of average molybdenum intake in the Japanese population. Journal of Nutritional Science and Vitaminology, 50, 404-409. doi:10.3177/jnsv.50.404

[17] Haddad, E.H., Berk, L.S., Kettering, J.D., Hubbard, R.W. and Peters, W.R. (1999) Dietary intake and biochemical, hematologic, and immune status of vegans compared with nonvegetarians. American Journal of Clinical Nutrition, 70, 586S-593S.

[18] Ogirima, M., Sasaki, K., Ioku, K., Kajiwara, N., Okada, M. and Okuda, T. (2004) Nutritional assessment of plantbased diet from the aspect of serum trace elements: zinc, copper and selenium. Trace Nutrient Research, 21, 149152 (in Japanese).

[19] Yoshida, M. and Yasumoto, K. (1987) Selenium contents of rice grown at various sites in Japan. Journal of Food Composition and Analysis, 1, 71-75. doi:10.1016/0889-1575(87)90013-5

[20] Ikebe, K., Tanaka, Y. and Tanaka, R. (1987) Daily intakes of 15 metals according to the duplicate portion studies. Japanese Journal of Food Hygiene, 29, 52-57 (in Japanese). doi:10.3358/shokueishi.29.52

[21] Miyazaki, Y., Koyama, H., Sasada, Y., Satoh, H., Nojiri, M. and Suzuki, S. (2004) Dietary habits and selenium intake of residents in mountain and coastal communities in Japan. Journal of Nutritional Science and Vitaminology, 50, 309-319. doi:10.3177/jnsv.50.309

[22] Yoshida, M. and Yasumoto, K. (1988) Selenium content in wheat and soybean products consumed in Japan. Journal of Japanese Society of Nutrition and Food Science, 41, 320-323 (in Japanese).

doi: $10.4327 /$ jsnfs. 41.320 
[23] Katamine, S., Mamiya, Y., Sekimoto, K., Hoshino, N., Totsuka, K., Naruse, U., Watabe, A., Sugiyama, R. and Suzuki, M. (1986) Iodine content of various meals currently consumed by urban Japanese. Journal of Nutritional Science and Vitaminology, 32, 487-495. doi:10.3177/jnsv.32.487

[24] Berkow, S.E. and Barnard, N.D. (2005) Blood pressure regulation and vegetarian diets. Nutrition Reviews, 63, 1-8. doi:10.1111/j.1753-4887.2005.tb00104.x 